\title{
Content and Classification of Clinical Trials at a University Hospital in Japan
}

\author{
Tetsuya NAKAMURA, ${ }^{1}$ MD, Koujirou YAMAMOTO, ${ }^{1,2} \mathrm{PhD}$, \\ Ryozo NAGAI, ${ }^{3} \mathrm{MD}$, and Ryuya HorIUCHI, ${ }^{1,2} \mathrm{PhD}$
}

\begin{abstract}
SUMMARY
"Standards on the Implementation of Clinical Trials on Drugs (New GCP)" is a Japanese government policy established in April 1998 with the aim of satisfying scientific and ethical requirements for industry-sponsored research, i.e., registration-directed clinical trials and clinical trials intended to support reexamination or reevaluation applications. Since then, efforts for more effective implementation of clinical trials have been promoted, including establishment of a system to invite more active participation of subjects in clinical trials and improvement of a network of medical institutions conducting clinical trials. These efforts should help to reactivate clinical trials in Japan, which reportedly have become stagnant. Although the New GCP addresses the quality of industry-sponsored clinical trials, investigators also construct study protocols without industry involvement. We reviewed clinical trials submitted by investigators at Gunma University Hospital to institutional review boards (IRBs) from June 1999 to February 2002. Ten clinical research coordinators contributed to the present survey. A total of 151 investigator-initiated clinical trials reviewed included a wide variety of content; and investigators from many institutions and organizations conducted trials. Most of the ethical guidelines for approving proposed trials represented the provisions of the Declaration of Helsinki. However, additional guidelines prepared by the Japanese Ministry of Health, Labour and Welfare were also helpful. Development of a support system for clinical trails requires the contribution of clinical research coordinators. Flexible management and careful attention to both the protocol and its execution by the investigators were also important for promoting clinical trials on the basis of meticulous patient care. (Jpn Heart J 2003; 44: 235-242)
\end{abstract}

Key words: Clinical trial, Institutional review board, Clinical research coordinator

THE World Medical Association has developed the Declaration of Helsinki as a statement of ethical principles to provide guidance to physicians and other participants in medical research involving human subjects. ${ }^{1)}$ A physician conducting medical research has a duty to protect the life, health, privacy, and dignity of

From ${ }^{1}$ Clinical Investigation and Research Unit, Gunma University Hospital, ${ }^{2}$ Department of Clinical Pharmacology, Gunma University School of Medicine, Gunma, ${ }^{3}$ Deparment of Cardiovascular Medicine, University of Tokyo Graduate School of Medicine, Tokyo, Japan.

Address for correspondence; Tetsuya Nakamura, MD, Clinical Investigation and Research Unit, Gunma University Hospital Maebashi, Gunma 371-8511 Japan.

Received for publication July 17, 2002.

Revised and accepted August 26, 2002. 
human subjects. According to the Declaration of Helsinki, the design and performance of each experimental procedure involving human subjects should be clearly formulated in an experimental protocol. This protocol should be submitted for consideration, comment, guidance, and where appropriate, approval to a specially appointed ethics review committee independent of the investigator and the sponsor, with no other kind of undue influence.

The Japanese Ministry of Health, Labour and Welfare enacted "Standards on the Implementation of Clinical Trials on Drugs (New GCP)" in April 1998, aiming to satisfy many scientific and ethical requirements. ${ }^{2}$ According to these standards, the committee has the right to monitor ongoing trials. The researcher has an obligation to provide monitoring information to the committee, especially regarding serious adverse events. The researcher should also submit to the committee all information regarding funding, sponsors, institutional affiliations, other potential conflicts of interest, and incentives for subjects.

Many institutional settings for clinical trials in Japan long have fallen short of such standards. Investigators often construct study protocols without corporate sponsor oversight. Many hospitals have not established the administrative framework to fulfill the criteria of the Declaration of Helsinki or the New GCP, leading to delays in developing new drugs or techniques and the risk of violating the rights of the patients. To date, no report has examined the structure and content of investigator-initiated clinical trials submitted to IRBs in Japan. The Clinical Investigation and Research Unit (CIRU) of Gunma University Hospital has been a particularly successful system for the support and development of clinical trials. In this paper, we examined investigator-initiated clinical trials submitted to Gunma University Hospitel IRB and supported by the CIRU at Gunma University Hospital.

\section{Methods}

Investigator-initiated clinical trials submitted to the IRB at Gunma University Hospital from June 1999 to February 2002 were classified. The CIRU consists of physicians from a variety of specialties, a pharmacist, a registered nurse, and a clinical pharmacologist. The inspection sector of the CIRU conducts an initial review of a protocol. Clinical research coordinators also attend. After the principal investigator explains the protocol, the members inquire about details of the protocol with respect to feasibility, safety, and efficacy. This initial review process covers scientific, ethical, and feasibility issues of each protocol, and typically requires 60 to 90 minutes. The results of the initial hearing are reported to the IRB by a member of the CIRU inspection sector. During the process, all the 
study protocols are characterized according to a classification system originally developed by the CIRU.

The classification includes four categories: subject, objectives, content of the study, and ethical guidelines.

The "subject" category refers to the main person or entity conducting the study. The "subject" category includes protocols conducted solely by investigators at Gunma University Hospital; protocols conducted by government bodies such as the Ministry of Education, Culture, Sports, Science and Technology, or the Ministry of Health, Labour and Welfare; protocols conducted by investigators at Gunma University in cooperation with investigators at other hospitals as multicenter trials; protocols conducted by academic societies or other nongovernmental organizations; and protocols conducted mainly by investigators at other hospitals in this region in cooperation with investigators at Gunma University Hospital.

The "Objectives" category includes study aims to develop new diagnostic or therapeutic procedures; to investigate etiology, pathogenesis and disease mechanisms; to further social medicine and/or education; to conduct an epidemiologic survey; and to obtain approval for a new diagnostic or therapeutic modality from the Ministry of Health, Labour and Welfare.

The "Content" category includes transplantation (bone marrow transplantation, amnion transplantation, and others including autotransplantation); new medical or surgical treatment procedures; analysis of cells or tissues obtained by biopsy or other surgery; psychological intervention; clinical effects of chemicals; clinical effects of food, cosmetics, or nondrug therapeutic preparations; examination of blood, urine, or sputum; assessment of administrative procedures and new clinical effects of approved drugs; and the clinical effects of investigational new drugs.

The "Ethical guidelines" category is concerned with the protocol which was reviewed according to the Declaration of Helsinki or "Standards on the Implementation of Clinical Trials on Drugs (New GCP)"; according to governmental guidelines for genetic research (germ-line mutation or polymorphism); according to guidelines for clinical gene therapy; according to guidelines for the use of tissues or organs obtained by biopsy or other surgical procedure; according to guidelines for epidemiologic surveys; reviewed in terms of the use of tissues or organs obtained from cadavers.

\section{RESULTS}

A total of 151 protocols were reviewed. Subject categories included 108 protocols conducted solely by investigators at Gunma University Hospital; 10 
protocols conducted by government bodies; 11 protocols conducted by investigators of Gunma University in cooperation with clinical investigators at other hospitals as a multicenter trial, 21 protocols conducted by academic or other organizations; and 1 protocol conducted by another hospital in the local area in cooperation with investigators at Gunma University Hospital (Figure 1).

The objectives included 15 protocols for the development of new diagnostic procedures; 87 for the development of new therapeutic methods; 25 for the investigation of etiology, pathogenesis and mechanisms; 1 for social medicine and educational reasons; 6 for obtaining epidemiologic information by survey; 13 for obtaining government approval of a new therapeutic modality; and 4 for obtaining approval for a new diagnostic measure (Figure 2).

The content categories included 8 protocols concerning transplantation; 14 new surgical equipment or procedures such as laser photocoagulation or thoracoscopic or laparoscopic surgical procedures; 14 new medical equipment or procedures such as radiofrequency thermal ablation; 14 investigations of tissues obtained for diagnosis or treatments; 2 psychological interventions; 8 clinical effects of chemicals; 6 clinical effects of food, cosmetics, and nondrug therapeutic preparations; 20 examinations of blood, urine, and sputum; 57 new administrative methods or clinical effects of approved drugs; 3 clinical effects of

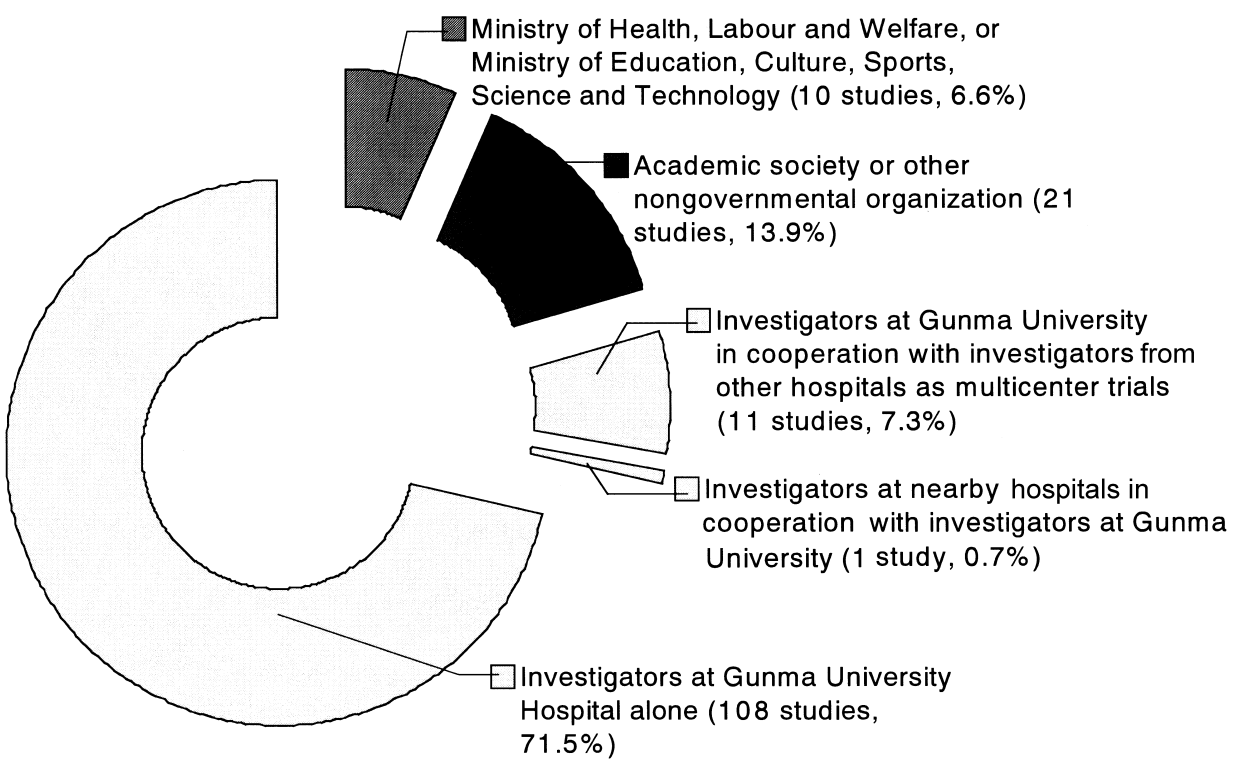

Figure 1. Subject categories. 


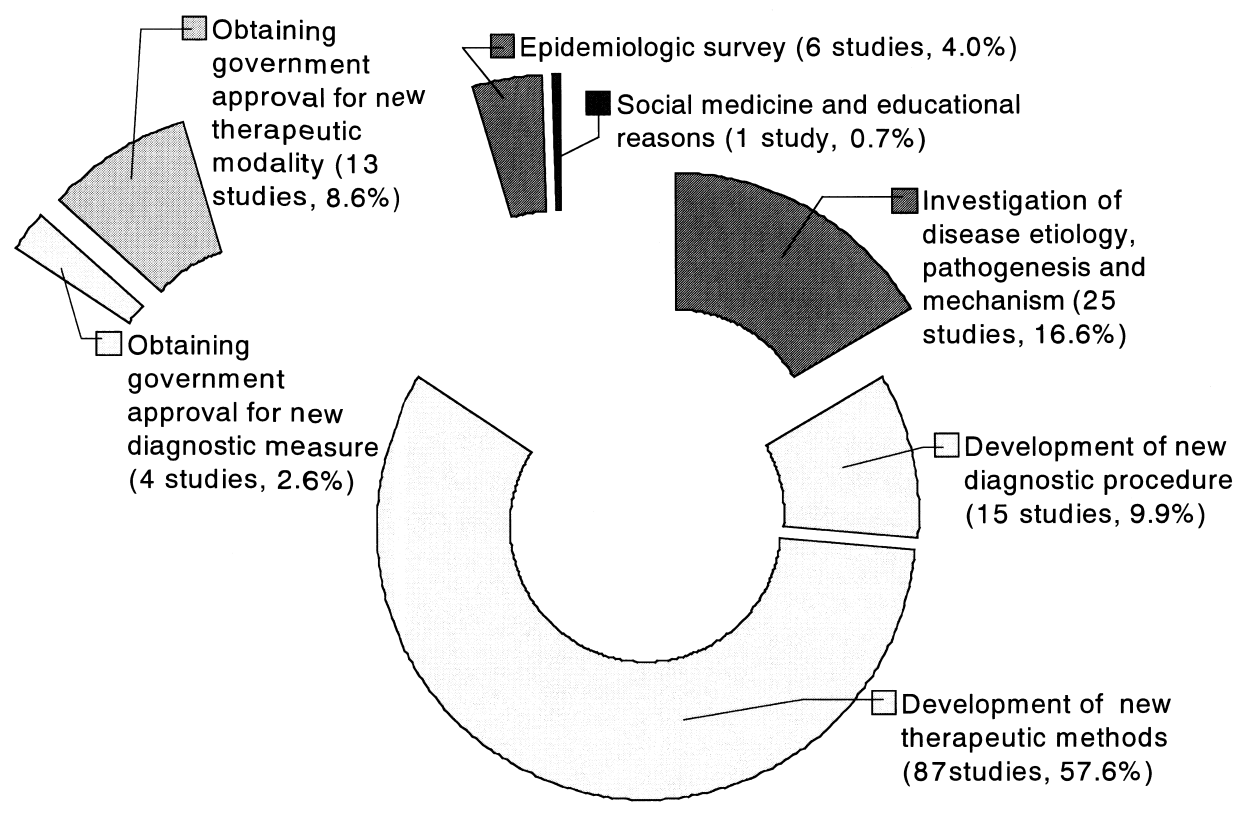

Figure 2. Objective categories.

investigational new drugs; and 5 observational studies, database searches or questionnaire surveys (Figure 3).

The ethical guideline category included 113 protocols reviewed according to the Declaration of Helsinki or the New GCP; 10 protocols reviewed using guidelines for genetic research; no protocols reviewed in terms of clinical gene therapy guidelines; 19 protocols reviewed using guidelines for use of excised or resected tissues or organs; 3 protocols reviewed in terms of somatic mutations emerging in diseased tissues or organs not inherited by offspring; 5 protocols reviewed using guidelines for epidemiologic surveys; and 1 protocol reviewed in terms of the use of cadaveric tissue (Figure 4). 


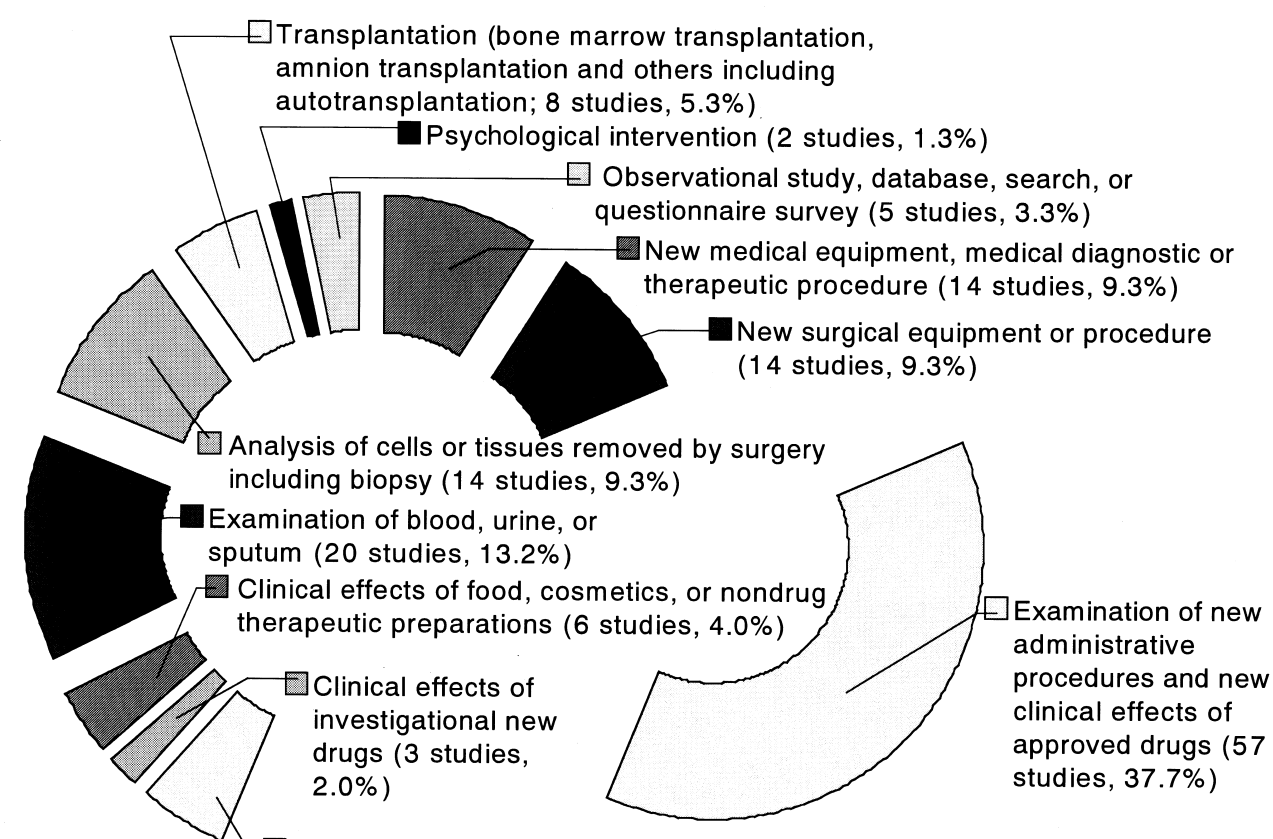

$\square$ Clinical effect of chemicals (8 studies, $5.3 \%$ )

Figure 3. Content categories.

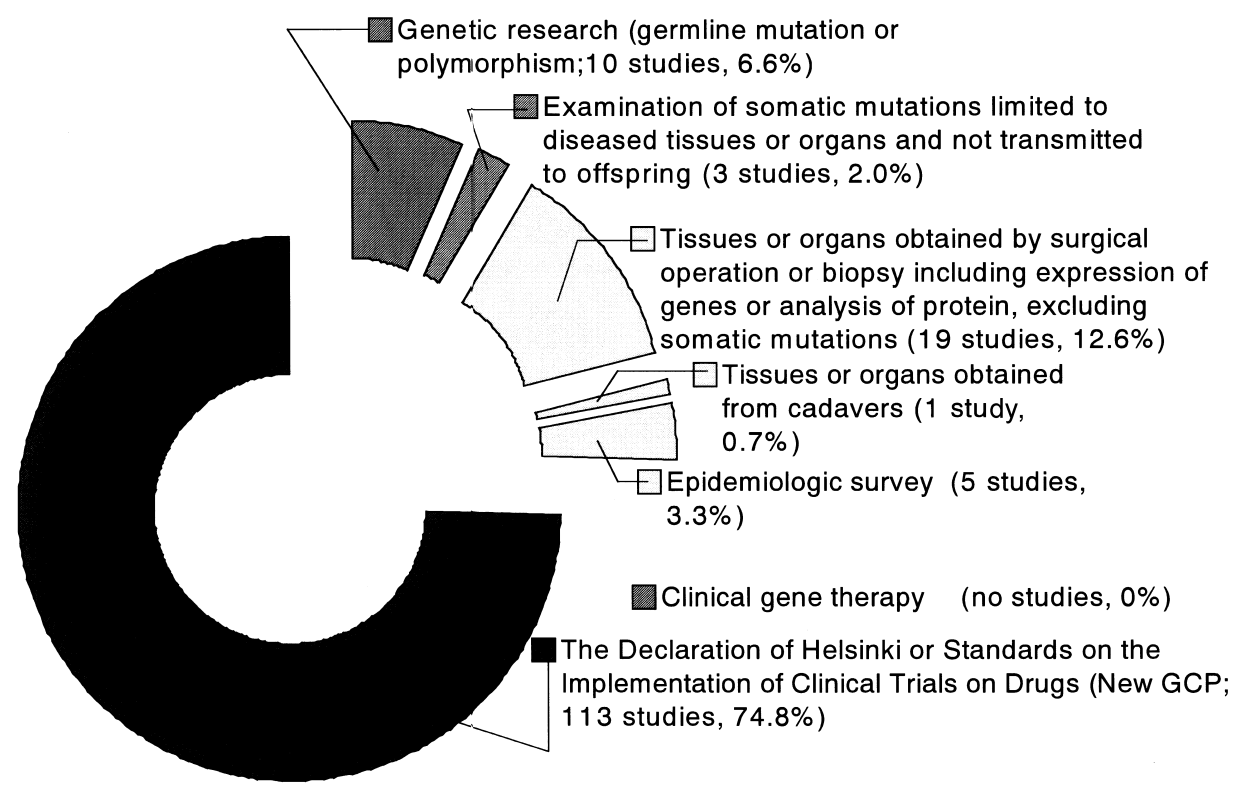

Figure 4. Ethical guidelines categories. 


\section{DISCUSSION}

In the present survey we categorized clinical studies undertaken at Gunma University Hospital. Large numbers of clinical studies are conducted in Japanese university hospitals. These studies cover a wide range of subjects including psychological intervention and consumer products. Topic-specific ethical guidelines are most appropriate for assessing these various clinical study protocols. The Ministry of Health, Labour and Welfare provides ethics guidelines for genetic research, clinical gene therapy, the use of tissues removed for diagnosis or treatment, and epidemiologic surveys. The Declaration of Helsinki and the New GCP represent the basic and most important rules for reviewing ethical points of clinical study protocols. In some clinical trials, however, obtaining informed consent is impractical or impossible. One example would be a national survey characterizing bacteria obtained from clinical specimens, where data are obtained from databases at hospitals. Guidelines for epidemiologic study indicate which protocols do not require informed consent. Guidelines for epidemiologic surveys state that patients should be notified using posters or other forms of announcements when a clinical survey is being performed in a hospital. Such guidelines act to promote clinical study because investigators and IRB members encounter fewer ethical ambiguities, accelerating the review process. Thus, classification of clinical research is necessary to apply the ethical guidelines that are best suited to the type of study.

The "Subjects" conducting the studies were mainly investigators at Gunma University Hospital. Multicenter trials also were included. More institutions need to be equipped with support systems to facilitate clinical trials. Academic societies also are important in organizing and promoting clinical trials. In the present survey, only a few clinical studies were conducted by either government bodies or academic societies.

The objectives of clinical trials should be reviewed carefully for appropriateness and attainability. In the present survey, the development of new treatments and diagnostic modalities was the most common objective, though a few studies were simply educational or observational. Ultimately, the significance of a clinical study involves its long-term impact on clinical practice and on medical research.

The New GCP, used as a regulation covering investigator-initiated clinical trials at Gunma University Hospital, is a system of shared responsibility in which the IRB together with sponsors, monitors, clinical investigators, and regulators all participate in protecting the rights and welfare of the human subjects. ${ }^{2)}$ The structure, function, operation, and authority of the IRB have been addressed by International Conference of Harmonization E5) and "Standards on the Imple- 
mentation of Clinical Trials on Drugs (New GCP)" set forth by the Ministry of Health, Labour and Welfare in 1998. An IRB must adopt standard operating procedures for conducting initial and continuing review of the research, reporting its actions to the investigator and institution, determining which projects require review more often than annually, ensuring prompt reporting of changes in the research to the IRB, ensuring that changes in the research are not initiated without IRB review, and requiring that informed consent be obtained where applicable.

Clinical research coordinators are important for ensuring adherence to standard operating procedures. Clinical research coordinators should check the progress and status of the study protocol after approval by the IRB and confirm that clinical trials are performed ethically. A total of 10 clinical research coordinators contributed to the present survey. Clinical research coordinators visit each principal investigator to monitor the number of patients already enrolled in the study, occurrence of significant adverse effects, changes in members of the research group, and changes in the protocols. The activities of clinical research coordinators help to promote the quality, safety, and ethical soundness of clinical research.

In summary, studies at university hospitals in Japan cover many fields and experimental designs. A support system for clinical trials including the contribution of clinical research coordinators, is essential to provide consistent yet flexible management while protecting patients and promoting investigation.

\section{REFERENCES}

1. Lewis JA, Jonsson B, Kreutz G, Sampaio C, van Zwieten-Boot B. Placebo-controlled trials and the Declaration of Helsinki. Lancet 2002; 359: 1337-40.

2. Fujiwara Y, Kobayashi K. Oncology drug clinical development and approval in Japan: the role of the pharmaceuticals and medical devices evaluation center (PMDEC). Crit Rev Oncol Hemat 2002; 42: 145-55.

3. Dixon JR Jr. The International Conference on Harmonization Good Clinical Practice Guideline. Qual Assur 1998; 6: 65-74. 